\title{
Total Enantioselective Synthesis of (-)-Cytisine
}

Bruno Danieli, Giordano Lesma,* Daniele Passarella, Alessandro Sacchetti, Alessandra Silvani,* Andrea Virdis

Dipartimento di Chimica Organica e Industriale e Centro Interdisciplinare Studi biomolecolari e applicazioni Industriali (CISI), Università degli Studi di Milano, via G. Venezian 21, 20133 Milano

\section{Supporting Information Available}

(3S,5R) (+)-3-Acetoxymethyl-5-formyl-piperidine-1-carboxylic acid benzyl ester 5 was prepared according to ref. 12 .

(3S,5R, 1'S) (-)-3-Acetoxymethyl-5-(1'-hydroxy-but-3'-enyl)-piperidine-1-carboxylic acid benzyl ester 6a

To a suspension of (-)- $B$-methoxydiisopinocampheylborane $(1.2 \mathrm{~g}, 3.8 \mathrm{mmol})$ in ether $(18 \mathrm{~mL})$ under nitrogen at $-78^{\circ} \mathrm{C}$, a solution of allylmagnesium bromide $(1.0 \mathrm{M}$ in ether, $3.8 \mathrm{~mL}, 3.8 \mathrm{mmol})$ was added dropwise. The mixture was stirred for $15 \mathrm{~min}$ at $-78^{\circ} \mathrm{C}$ and for $1 \mathrm{~h}$ at room temperature, and then it was recooled to $-78^{\circ} \mathrm{C}$. A solution of $5(933 \mathrm{mg}, 2.9$ mmol) in ether $(10 \mathrm{~mL})$ was added, and the mixture was stirred for $2 \mathrm{~h}$ at $-78^{\circ} \mathrm{C}$ and for $2 \mathrm{~h}$ at room temperature. A $3 \mathrm{~N}$ sodium hydroxide solution $(15 \mathrm{~mL})$ was added, followed by $30 \%$ hydrogen peroxide solution $(15 \mathrm{~mL})$, and the mixture was heated for $3 \mathrm{~h}$. After cooling, the mixture was diluted with ether $(40 \mathrm{~mL})$ and it was washed with water $(30 \mathrm{~mL})$ and brine $(30 \mathrm{~mL})$. The aqueous washings were extracted three times with diethyl ether $(40 \mathrm{~mL})$, and the combined ethereal extracts were dried over $\mathrm{Na}_{2} \mathrm{SO}_{4}$. Removal of the solvent under reduced pressure was followed by flash chromatography of the residue on silica gel (ethyl acetate/hexane 1:5), to yield $801 \mathrm{mg}(76 \%)$ of $\mathbf{6 a}$ as a colorless oil: $\mathrm{R}_{f}$ (ethyl acetate/hexane 1:5) 0.30; $[\alpha]^{25}-1.4\left(c\right.$ 1, $\left.\mathrm{CHCl}_{3}\right) ;{ }^{1} \mathrm{H}$ NMR $\left(300 \mathrm{MHz}, \mathrm{CDCl}_{3}\right) \delta_{\mathrm{H}} 7.35(5 \mathrm{H}, \mathrm{br}, \mathrm{s}), 5.88-5.70$ $(1 \mathrm{H}, \mathrm{m}), 5.18-5.10(2 \mathrm{H}, \mathrm{m}), 5.12(2 \mathrm{H}, \mathrm{s}), 4.40(1 \mathrm{H}, \mathrm{d}, J=13.3 \mathrm{~Hz}), 4.25(1 \mathrm{H}, \mathrm{d}, J=13.3 \mathrm{~Hz}), 4.00(1 \mathrm{H}, \mathrm{dd}, J=11.7$, $6.7 \mathrm{~Hz}), 3.85(1 \mathrm{H}, \mathrm{dd}, J=11.7,7.3 \mathrm{~Hz}), 3.50-3.40(1 \mathrm{H}, \mathrm{m}), 2.55(1 \mathrm{H}, \mathrm{t}, J=12.1 \mathrm{~Hz}), 2.40(1 \mathrm{H}, \mathrm{t}, J=12.6 \mathrm{~Hz}), 2.30-$ $2.10(2 \mathrm{H}, \mathrm{m}), 2.00(3 \mathrm{H}, \mathrm{s}), 1.90-1.80(1 \mathrm{H}, \mathrm{m}), 1.75(1 \mathrm{H}, \mathrm{d}, J=12.2 \mathrm{~Hz}), 1.65-1.50(1 \mathrm{H}, \mathrm{m}), 1.08(1 \mathrm{H}, \mathrm{q}, J=12.2 \mathrm{~Hz})$;

${ }^{13} \mathrm{C}$ NMR $\left(75.4 \mathrm{MHz}, \mathrm{CDCl}_{3}\right) \delta_{\mathrm{C}} 170.8,155.1,136.8,134.2,127.1-130.2(5 \mathrm{C}), 118.7,71.7,67.1,66.6,46.8,40.8,39.1$, 35.4, 29.7, 20.8; HRMS calcd for $\mathrm{C}_{20} \mathrm{H}_{27} \mathrm{NO}_{5}$ : 361.4419. Found: 361.4431.

(3S,5R, 1'R) (-)-3-Acetoxymethyl-5-(1'-hydroxy-but-3'-enyl)-piperidine-1-carboxylic acid benzyl ester 6b

6b was prepared following the procedure described for $\mathbf{6 a}$, using (+)- $B$-methoxydiisopinocampheylborane in place of the (-)-antipode (67\% yield). $\mathrm{R}_{f}$ (ethyl acetate/hexane 1:5) $0.30 ;[\alpha]^{25}-1.6\left(c 1, \mathrm{CHCl}_{3}\right) ;{ }^{1} \mathrm{H} \mathrm{NMR}\left(300 \mathrm{MHz}, \mathrm{CDCl}_{3}\right)$ $\delta_{\mathrm{H}} 7.35(5 \mathrm{H}, \mathrm{br}, \mathrm{s}), 5.88-5.75(1 \mathrm{H}, \mathrm{m}), 5.20-5.12(2 \mathrm{H}, \mathrm{m}), 5.15(2 \mathrm{H}, \mathrm{s}), 4.30(1 \mathrm{H}, \mathrm{d}, J=10.2 \mathrm{~Hz}), 4.20(1 \mathrm{H}, \mathrm{d}, J=12.2$ $\mathrm{Hz}), 4.04(1 \mathrm{H}, \mathrm{dd}, J=11.2,5.3 \mathrm{~Hz}), 3.91(1 \mathrm{H}, \mathrm{dd}, J=11.2,7.2 \mathrm{~Hz}), 3.58-3.50(1 \mathrm{H}, \mathrm{m}), 2.65(1 \mathrm{H}, \mathrm{t}, J=12.3 \mathrm{~Hz}), 2.47$ $(1 \mathrm{H}, \mathrm{t}, J=12.3 \mathrm{~Hz}), 2.34(1 \mathrm{H}, \mathrm{dt}, J=13.9,4.8 \mathrm{~Hz}), 2.22(1 \mathrm{H}, \mathrm{dt}, J=14.0,8.3 \mathrm{~Hz}), 2.05(3 \mathrm{H}, \mathrm{s}), 2.00(1 \mathrm{H}, \mathrm{d}, J=13.0$ $\mathrm{Hz}), 1.95-1.83(1 \mathrm{H}, \mathrm{m}), 1.70-1.58(1 \mathrm{H}, \mathrm{m}), 1.10(1 \mathrm{H}, \mathrm{q}, J=12.2 \mathrm{~Hz}) ;{ }^{13} \mathrm{C} \mathrm{NMR}\left(75.4 \mathrm{MHz}, \mathrm{CDCl}_{3}\right) \delta_{\mathrm{C}} 171.0,155.3$, 
136.8, 134.2, 128.5-127.8 (5C), 118.9, 71.6, 67.1, 66.5, 47.1, 46.8, 40.6, 39.3, 35.5, 28.3, 20.8; HRMS calcd for $\mathrm{C}_{20} \mathrm{H}_{27} \mathrm{NO}_{5}: 361.4419$. Found: 361.4428.

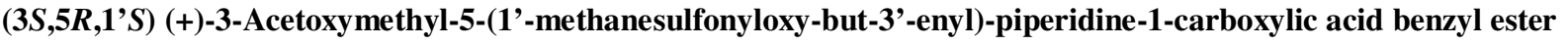
7

To a solution of $6 \mathbf{a}(843 \mathrm{mg}, 2.3 \mathrm{mmol})$ in anhydrous $\mathrm{CH}_{2} \mathrm{Cl}_{2}(20 \mathrm{~mL})$, under nitrogen atmosphere at $0^{\circ} \mathrm{C}, \mathrm{Et}_{3} \mathrm{~N}(1.6$ mL, $11.7 \mathrm{mmol}$ d $0.7 \mathrm{~g} / \mathrm{ml})$, DMAP (28 mg, $0.23 \mathrm{mmol})$ were added and methanesulfonyl chloride $(270 \mu \mathrm{L}, 3.5 \mathrm{mmol}$, d $1.5 \mathrm{~g} / \mathrm{mL}$ ) was slowly dropped. The mixture was stirred for $1.5 \mathrm{~h}$ at room temperature, then washed with $5 \%$ aq. $\mathrm{H}_{3} \mathrm{PO}_{4}$ (2 $\times 20 \mathrm{~mL}$ ). The organic layer was dried over $\mathrm{Na}_{2} \mathrm{SO}_{4}$ and concentrated to give $1 \mathrm{~g}(99 \%)$ of 7, as an oil: $\mathrm{R}_{f}$ (ethyl acetate/hexane 1:1) 0.40; $[\alpha]^{25}{ }_{\mathrm{D}}+3.2\left(c 1, \mathrm{CHCl}_{3}\right) ;{ }^{1} \mathrm{H} \mathrm{NMR}\left(300 \mathrm{MHz}, \mathrm{CDCl}_{3}\right) \delta_{\mathrm{H}} 7.35(5 \mathrm{H}, \mathrm{br}, \mathrm{s}), 5.90-5.70$ $(1 \mathrm{H}, \mathrm{m}), 5.18-5.10(2 \mathrm{H}, \mathrm{m}), 5.12(2 \mathrm{H}, \mathrm{s}), 4.50-4.30(2 \mathrm{H}, \mathrm{m}), 4.00(1 \mathrm{H}, \mathrm{dd}, J=15.4,6.1 \mathrm{~Hz}), 3.88(1 \mathrm{H}, \mathrm{dd}, J=15.4,6.3$ $\mathrm{Hz}), 3.55-3.40(1 \mathrm{H}, \mathrm{m}), 3.00(3 \mathrm{H}, \mathrm{s}), 2.55(1 \mathrm{H}, \mathrm{t}, J=12.8 \mathrm{~Hz}), 2.45(1 \mathrm{H}, \mathrm{t}, J=12.8 \mathrm{~Hz}), 2.40-2.10(2 \mathrm{H}, \mathrm{m}), 2.05(3 \mathrm{H}$, s), 2.00-1.90 $(1 \mathrm{H}, \mathrm{m}), 1.90-1.80(1 \mathrm{H}, \mathrm{m}), 1.60-1.50(1 \mathrm{H}, \mathrm{br}), 1.18(1 \mathrm{H}, \mathrm{q}, J=12.8 \mathrm{~Hz})$; HRMS calcd for $\mathrm{C}_{21} \mathrm{H}_{29} \mathrm{NO}_{7} \mathrm{~S}$ : 439.5318. Found: 439.5328 .

\section{$(3 S, 5 R, 1 ' R)$ (-)-3-Acetoxymethyl-5-(1'-azido-but-3'-enyl)-piperidine-1-carboxylic acid benzyl ester 8}

To a solution of 7 (1g, $2.3 \mathrm{mmol})$ in anhydrous DMF (30 mL), NaN 3 (224 mg, $3.4 \mathrm{mmol})$ was added under nitrogen atmosphere and the mixture was stirred at $80^{\circ} \mathrm{C}$ for $2 \mathrm{~h}$. The suspension was diluted with diethyl ether $(30 \mathrm{~mL})$ and added to water $(30 \mathrm{~mL})$. The aqueous layer was extracted with diethyl ether $(3 \mathrm{x} 20 \mathrm{~mL})$. The combined organic layers were washed with brine and dried over $\mathrm{Na}_{2} \mathrm{SO}_{4}$. Removal of the solvent in vacuum gave an oily residue which was purified by flash chromatography on silica gel (ethyl acetate/hexane 2:5), affording $765 \mathrm{mg}\left(87 \%\right.$ ) of $\mathbf{8}$, as an oil: $\mathrm{R}_{f}$ (ethyl acetate/hexane 2:5) 0.23; $[\alpha]^{25}$ - $29.0\left(c 1, \mathrm{CHCl}_{3}\right) ;{ }^{1} \mathrm{H}$ NMR $\left(300 \mathrm{MHz}, \mathrm{CDCl}_{3}\right) \delta_{\mathrm{H}} 7.35(5 \mathrm{H}, \mathrm{br}, \mathrm{s}), 5.80-5.55$ $(1 \mathrm{H}, \mathrm{m}), 5.17-5.10(2 \mathrm{H}, \mathrm{m}), 5.12(2 \mathrm{H}, \mathrm{s}), 4.40-4.15(2 \mathrm{H}, \mathrm{m}), 4.00(1 \mathrm{H}, \mathrm{dd}, J=12.1,6.1 \mathrm{~Hz}), 3.90(1 \mathrm{H}, \mathrm{dd}, J=12.1,7.0$ Hz), 3.34-3.25 (1H, m), $2.65(1 \mathrm{H}, \mathrm{t}, \mathrm{J}=12.3 \mathrm{~Hz}), 2.55(1 \mathrm{H}, \mathrm{t}, \mathrm{J}=12.3 \mathrm{~Hz}), 2.45-2.34$ (2H, m), 2.00 (3H, s), $1.90-1.65$ $(2 \mathrm{H}, \mathrm{m}), 1.60-1.46(1 \mathrm{H}, \mathrm{m}), 1.10(1 \mathrm{H}, \mathrm{q}, \mathrm{J}=13.1 \mathrm{~Hz}) ;{ }^{13} \mathrm{C}$ NMR $\left(75.4 \mathrm{MHz}, \mathrm{CDCl}_{3}\right) \delta_{\mathrm{C}} 170.8,155.1,133.2,127.0-$ 130.0 (5C), 118.7, 68.1, 66.2, 64.3, 47.1, 45.6, 39.2, 35.5, 28.8, 20.6; HRMS calcd for $\mathrm{C}_{20} \mathrm{H}_{26} \mathrm{~N}_{4} \mathrm{O}_{4}$ : 386.4546. Found: 386.4561 .

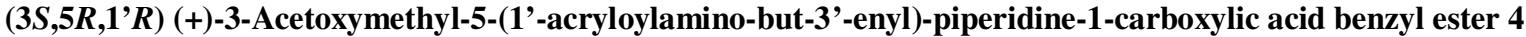

To a solution of $8(141 \mathrm{mg}, 0.36 \mathrm{mmol})$ in THF ( $5 \mathrm{~mL})$, triphenylphosphine (96 mg, $0.36 \mathrm{mmol})$ was added. After the reaction was complete (controlled by TLC), $\mathrm{H}_{2} \mathrm{O}(330 \mu \mathrm{L}, 18.3 \mathrm{mmol})$ was added. The mixture was stirred overnight, then concentrated in vacuum and the water removed by azeotropic distillation with toluene. The crude amine was dissolved in anhydrous $\mathrm{CH}_{2} \mathrm{Cl}_{2}(6 \mathrm{~mL})$ under nitrogen atmosphere and cooled to $0^{\circ} \mathrm{C}$. To this stirred solution $\mathrm{Et}_{3} \mathrm{~N}(112$ $\mu \mathrm{L}, 0.80 \mathrm{mmol}, \mathrm{d} 0.7 \mathrm{~g} / \mathrm{mL}$ ) and DMAP (4 mg, $0.04 \mathrm{mmol})$ were added and acryloyl chloride (39 $\mu \mathrm{L}, 0.47 \mathrm{mmol}, \mathrm{d} 1.1$ $\mathrm{g} / \mathrm{mL}$ ) was slowly dropped. After stirring at $0^{\circ} \mathrm{C}$ for $30 \mathrm{~min}$ and at room temperature for $4 \mathrm{~h}$, the reaction mixture was washed with $1 \mathrm{~N} \mathrm{HCl}$ and then with $5 \%$ aq. $\mathrm{NaHCO}_{3}$. Removal of the solvent under reduced pressure was followed by flash chromatography on silica gel (ethyl acetate/hexane 3:2) to yield $89 \mathrm{mg}$ (59\%, over two steps) of $\mathbf{4}$, as an oil: $\mathrm{R}_{f}$ 
(ethyl acetate/hexane 3:2) 0.29; $[\alpha]^{25}{ }_{\mathrm{D}}+8.0\left(c 1, \mathrm{CHCl}_{3}\right) ;{ }^{1} \mathrm{H} \mathrm{NMR}\left(300 \mathrm{MHz}, \mathrm{CDCl}_{3}\right) \delta_{\mathrm{H}} 7.30(5 \mathrm{H}, \mathrm{br}, \mathrm{s}), 6.24(1 \mathrm{H}$, $\mathrm{dd}, J=16.0,5.0 \mathrm{~Hz}), 6.06(1 \mathrm{H}, \mathrm{dd}, J=16.0,10.0 \mathrm{~Hz}), 5.81-5.65(1 \mathrm{H}, \mathrm{m}), 5.63(1 \mathrm{H}, \mathrm{dd}, J=10.3,5.0 \mathrm{~Hz}), 5.35(1 \mathrm{H}, \mathrm{br}$, $\mathrm{d}, J=9.4 \mathrm{~Hz}), 5.17-5.02(2 \mathrm{H}, \mathrm{m}), 5.10(2 \mathrm{H}, \mathrm{s}), 4.36-4.18(2 \mathrm{H}, \mathrm{m}), 4.06-3.95(1 \mathrm{H}, \mathrm{m}), 3.86(1 \mathrm{H}, \mathrm{dd}, J=16.1,7.0 \mathrm{~Hz})$, $3.83(1 \mathrm{H}, \mathrm{dd}, J=11.1,7.1 \mathrm{~Hz}), 2.55-2.30(2 \mathrm{H}, \mathrm{m}), 2.26-2.13(2 \mathrm{H}, \mathrm{m}), 2.01(3 \mathrm{H}, \mathrm{s}), 1.92-1.78(1 \mathrm{H}, \mathrm{m}), 1.72-1.55(2 \mathrm{H}$, m), $0.92(1 \mathrm{H}, \mathrm{q}, \mathrm{J}=12.8 \mathrm{~Hz}) ;{ }^{13} \mathrm{C}$ NMR $\left(75.4 \mathrm{MHz}, \mathrm{CDCl}_{3}\right) \delta_{\mathrm{C}} 170.8,165.3,155.1,133.4,130.6,126.0-128.2(5 \mathrm{C})$, 118.5, 67.2, 66.3, 50.6, 47.1, 46.6, 39.0, 36.4, 35.5, 29.7, 20.8; HRMS calcd for $\mathrm{C}_{23} \mathrm{H}_{30} \mathrm{~N}_{2} \mathrm{O}_{5}$ : 414.5060. Found: 414.5072 .

\section{$\left(2 R, 3^{\prime} R, 5^{\prime} S\right)(+)-5$ '-Acetoxymethyl-6-oxo-1,2,3,6,3',4',5',6'-octahydro-2'H-[2,3']-bipyridinyl-1'- carboxylic acid benzyl ester 3}

To a stirred solution of $4(247 \mathrm{mg}, 0.6 \mathrm{mmol})$ in $\mathrm{CH}_{2} \mathrm{Cl}_{2}(10 \mathrm{~mL}) \mathrm{Cl}_{2} \mathrm{Ru}(\mathrm{CHPh})\left(\mathrm{P}(c \text {-hexyl })_{3}\right)_{2}$ (24 mg, $0.03 \mathrm{mmol}$, 5\% mol) was added under nitrogen atmosphere. The mixture was refluxed for $12 \mathrm{~h}$. DMSO (106 $\mu \mathrm{L}, 1.5 \mathrm{mmol}, \mathrm{d} 1.1 \mathrm{~g} / \mathrm{mL})$ was added and the mixture was stirred overnight at room temperature. The solvent was removed in vacuum and the residue was purified by flash chromatography on silica gel (ethyl acetate), to yield $183 \mathrm{mg}$ (79\%) of $\mathbf{3}$, as an oil: $\mathrm{R}_{f}$ (ethyl acetate) $0.15 ;[\alpha]^{25}+8.4\left(c 1, \mathrm{CHCl}_{3}\right) ;{ }^{1} \mathrm{H} \mathrm{NMR}\left(300 \mathrm{MHz}, \mathrm{CDCl}_{3}\right) \delta_{\mathrm{H}} 7.35(5 \mathrm{H}, \mathrm{br}, \mathrm{s}), 6.62(1 \mathrm{H}, \mathrm{dt}, J=11.6$, $2.9 \mathrm{~Hz}), 6.26(1 \mathrm{H}, \mathrm{s}), 5.91(1 \mathrm{H}, \mathrm{d}, J=9.9 \mathrm{~Hz}), 5.12(2 \mathrm{H}, \mathrm{s}), 4.38-4.16(2 \mathrm{H}, \mathrm{m}), 4.04(1 \mathrm{H}, \mathrm{dd}, J=11.1,5.8 \mathrm{~Hz}), 3.87$ $(1 \mathrm{H}, \mathrm{dd}, J=11.2,6.9 \mathrm{~Hz}), 3.56-3.41(1 \mathrm{H}, \mathrm{m}), 2.56-2.41(2 \mathrm{H}, \mathrm{m}), 2.40-2.23(2 \mathrm{H}, \mathrm{m}), 2.02(3 \mathrm{H}, \mathrm{s}), 1.85(1 \mathrm{H}, \mathrm{d}, J=11.6$ $\mathrm{Hz}), 1.81-1.68(2 \mathrm{H}, \mathrm{m}), 1.04(1 \mathrm{H}, \mathrm{q}, J=11.6 \mathrm{~Hz}) ;{ }^{13} \mathrm{C} \mathrm{NMR}\left(75.4 \mathrm{MHz}, \mathrm{CDCl}_{3}\right) \delta_{\mathrm{C}} 170.7,166.3,155.1,140.2,136.7$, 127.1-128.2 (5C), 124.6, 67.2, 66.1, 53.3, 46.3, 45.9, 39.9, 35.6, 29.9, 26.9, 20.6; HRMS calcd for $\mathrm{C}_{21} \mathrm{H}_{26} \mathrm{~N}_{2} \mathrm{O}_{5}$ : 386.4518. Found: 386.4523.

$\left(2 R, 3^{\prime} R, 5 ' S\right)(+)-5$ '-Hydroxymethyl-6-oxo-1,2,3,6,3',4',5',6'-octahydro-2'H-[2,3']-bipyridinyl-1'- carboxylic acid benzyl ester 9

To a solution of $3(205 \mathrm{mg}, 0.53 \mathrm{mmol})$ in THF $(2 \mathrm{~mL}) \mathrm{NaOH} 0.27 \mathrm{M}(3 \mathrm{~mL}, 0.81 \mathrm{mmol})$ was added and the mixture was stirred for $2 \mathrm{~h}$ at room temperature. After removing THF in vacuum, water is added, and the aqueous phase was extracted with ethyl acetate. The combined organic layers were dried over $\mathrm{Na}_{2} \mathrm{SO}_{4}$ and the solvent was removed under reduced pressure to yield $179 \mathrm{mg}(98 \%)$ of $\mathbf{9}$, as a foam: $\mathrm{R}_{f}$ (ethyl acetate) $0.08 ;[\alpha]^{25}{ }_{\mathrm{D}}+23.6\left(c 1, \mathrm{CHCl}_{3}\right) ;{ }^{1} \mathrm{H} \mathrm{NMR}$ $\left(300 \mathrm{MHz}, \mathrm{CDCl}_{3}\right) \delta_{\mathrm{H}} 7.32(5 \mathrm{H}, \mathrm{br}, \mathrm{s}), 6.61-6.51(1 \mathrm{H}, \mathrm{m}), 6.28-6.12(1 \mathrm{H}, \mathrm{br} \mathrm{s}), 5.89(1 \mathrm{H}, \mathrm{d}, J=7.8 \mathrm{~Hz}), 5.12(2 \mathrm{H}, \mathrm{s})$, 4.31-4.16 (2H, m), 3.55-3.37 (3H, m), 2.53-2.34 (2H, m), 2.35-2.19 (2H, m), $1.86(1 \mathrm{H}, \mathrm{d}, J=11.1 \mathrm{~Hz}), 1.79-1.62(2 \mathrm{H}$, $\mathrm{m}), 1.03(1 \mathrm{H}, \mathrm{q}, J=11.1 \mathrm{~Hz}) ;{ }^{13} \mathrm{C} \mathrm{NMR}\left(75.4 \mathrm{MHz}, \mathrm{CDCl}_{3}\right) \delta_{\mathrm{C}} 166.7,155.3,140.7,136.7,127.8-128.5(5 \mathrm{C}), 124.3$, 67.2, 64.9, 53.2, 46.3, 45.9, 39.8, 38.5, 29.6, 26.9; HRMS calcd for $\mathrm{C}_{19} \mathrm{H}_{24} \mathrm{~N}_{2} \mathrm{O}_{4}$ : 344.4141. Found: 344.4120.

\section{$\left(2 R, 3{ }^{\prime} R, 5 ' S\right)(+)-5$ '-Methanesulfonyloxymethyl-6-oxo-1,2,3,6,3',4',5',6'-octahydro-2'H-[2,3']bipyridinyl-1'- carboxylic acid benzyl ester 10}

To a solution of 9 (160 mg, $0.46 \mathrm{mmol}), \mathrm{Et}_{3} \mathrm{~N}(323 \mu \mathrm{L}, 2.32 \mathrm{mmol}, \mathrm{d} 0.73 \mathrm{~g} / \mathrm{mL})$, DMAP (10 mg, $\left.0.08 \mathrm{mmol}\right) \mathrm{in}$ anhydrous $\mathrm{CH}_{2} \mathrm{Cl}_{2}(10 \mathrm{~mL})$, methanesulfonyl chloride $(53 \mu \mathrm{L}, 0.69 \mathrm{mmol}, \mathrm{d} 1.5 \mathrm{~g} / \mathrm{mL})$ was added at $0^{\circ} \mathrm{C}$ under nitrogen atmosphere. The mixture was stirred for $1.5 \mathrm{~h}$ at room temperature, then washed with $5 \%$ aq. $\mathrm{H}_{3} \mathrm{PO}_{4}(2 \times 5 \mathrm{~mL})$. The 
organic layer was dried over $\mathrm{Na}_{2} \mathrm{SO}_{4}$ and concentrated under reduced pressure. Flash chromatography (ethyl acetate) gave $130 \mathrm{mg}(67 \%)$ of 10 , as an oil: $\mathrm{R}_{f}$ (ethyl acetate) $0.40 ;[\alpha]^{25}+3.2\left(c 1, \mathrm{CHCl}_{3}\right) ;{ }^{1} \mathrm{H} \mathrm{NMR}\left(300 \mathrm{MHz}, \mathrm{CDCl}_{3}\right) \delta_{\mathrm{H}}$ $7.35(5 \mathrm{H}, \mathrm{br}, \mathrm{s}), 6.61(1 \mathrm{H}, \mathrm{dt}, J=10.8,5.4 \mathrm{~Hz}), 6.50(1 \mathrm{H}, \mathrm{br}, \mathrm{s}), 6.12(1 \mathrm{H}, \mathrm{br}, \mathrm{s}), 5.93(1 \mathrm{H}, \mathrm{d}, J=10.8 \mathrm{~Hz}), 5.20(2 \mathrm{H}, \mathrm{s})$, $4.31(1 \mathrm{H}, \mathrm{d}, J=10.8 \mathrm{~Hz}), 4.23(1 \mathrm{H}, \mathrm{d}, J=10.8 \mathrm{~Hz}), 4.18-4.01(2 \mathrm{H}, \mathrm{m}), 3.51-3.42(1 \mathrm{H}, \mathrm{m}), 3.01(3 \mathrm{H}, \mathrm{s}), 2.65-2.36(2 \mathrm{H}$, $\mathrm{m}), 2.36-2.25(2 \mathrm{H}, \mathrm{m}), 1.93(1 \mathrm{H}, \mathrm{d}, J=12.4 \mathrm{~Hz}), 1.82-1.60(2 \mathrm{H}, \mathrm{m}), 1.10(1 \mathrm{H}, \mathrm{q}, J=12.4 \mathrm{~Hz}) ;{ }^{13} \mathrm{C} \mathrm{NMR}(75.4 \mathrm{MHz}$, $\left.\mathrm{CDCl}_{3}\right) \delta_{\mathrm{C}} 169.8,166.2,155.1,140.0,136.7,127.8-128.5$ (5C), 123.9, 70.8, 67.2, 53.3, 45.9, 46.3, 39.7, 37.5, 35.9, 29.5, 26.9; HRMS calcd for $\mathrm{C}_{20} \mathrm{H}_{26} \mathrm{~N}_{2} \mathrm{O}_{6} \mathrm{~S}: 422.5040$. Found: 422.5033.

\section{$(6 R, 7 R, 9 R)(+)-N-C b z-5,6-d i h y d r o-c y t i s i n e ~ 11$}

To a suspension of sodium hydride $(57 \mathrm{mg}, 0.88 \mathrm{mmol}, 80 \%$ dispersion in mineral oil) in anhydrous THF $(4 \mathrm{~mL})$ cooled to $0^{\circ} \mathrm{C}$, a solution of $\mathbf{1 0}(186 \mathrm{mg}, 0.44 \mathrm{mmol})$ in $\mathrm{THF}(4 \mathrm{~mL})$ was added. After 15 minutes at $0^{\circ} \mathrm{C}$, the reaction mixture was stirred at room temperature for $2 \mathrm{~h}$. A pH 7 phosphate buffer solution was added, THF was evaporated and the aqueous layer was extracted with ethyl acetate. The combined organic extracts were dried over $\mathrm{Na}_{2} \mathrm{SO}_{4}$ and concentrated to give $128 \mathrm{mg}(89 \%)$ of 11 , as an oil: $\mathrm{R}_{f}$ (ethyl acetate) $0.29 ;[\alpha]_{\mathrm{D}}+17.4\left(c 1, \mathrm{CHCl}_{3}\right) ;{ }^{1} \mathrm{H}$ NMR (300 $\left.\mathrm{MHz}, \mathrm{CDCl}_{3}\right) \delta_{\mathrm{H}} 7.30(5 \mathrm{H}, \mathrm{br}, \mathrm{s}), 6.53-6.47(1 \mathrm{H}, \mathrm{m}), 5.95(1 \mathrm{H}, \mathrm{dd}, J=10.1,2.4 \mathrm{~Hz}), 5.20(1 \mathrm{H}, \mathrm{d}, J=12.3 \mathrm{~Hz}), 5.10$ $(1 \mathrm{H}, \mathrm{d}, J=12.3 \mathrm{~Hz}), 4.39(1 \mathrm{H}, \mathrm{dd}, J=15.2,10.1 \mathrm{~Hz}), 4.19-3.98(2 \mathrm{H}, \mathrm{m}), 3.63-3.52(1 \mathrm{H}, \mathrm{m}), 3.00-2.91(2 \mathrm{H}, \mathrm{m}), 2.88$ $(1 \mathrm{H}, \mathrm{d}, J=15.2 \mathrm{~Hz}), 2.38-2.22(3 \mathrm{H}, \mathrm{m}), 1.92(1 \mathrm{H}, \mathrm{d}, J=13.5 \mathrm{~Hz}), 1.80(1 \mathrm{H}, \mathrm{br}, \mathrm{s}), 1.54(1 \mathrm{H}, \mathrm{d}, J=13.5 \mathrm{~Hz}),{ }^{13} \mathrm{C} \mathrm{NMR}$ (75.4 MHz, $\left.\mathrm{CDCl}_{3}\right) \delta_{\mathrm{C}} 170.7,169.1,155.1,138.1,137.5,127.8-128.5$ (5C), 125.7, 67.4, 55.9, 48.4, 49.9, 43.8, 34.5, 32.7, 25.7, 24.7; HRMS calcd for $\mathrm{C}_{19} \mathrm{H}_{22} \mathrm{~N}_{2} \mathrm{O}_{3}$ : 326.3988. Found: 326.3991 .

\section{(-)-N-Cbz-cytisine 12}

To a solution of DDQ (98 mg, $0.43 \mathrm{mmol})$ in dioxane $(4 \mathrm{~mL})$ a solution of $\mathbf{1 1}(140 \mathrm{mg}, 0.43 \mathrm{mmol})$ in $4 \mathrm{~mL}$ dioxane was added, under nitrogen atmosphere. The mixture was stirred and refluxed at $110^{\circ} \mathrm{C}$ for $4 \mathrm{~h}$. Removal of the solvent under reduced pressure was followed by flash chromatography (ethyl acetate/hexane 3:1) to yield $41 \mathrm{mg}$ (50\%) of 12, as an oil: $\mathrm{R}_{f}$ (ethyl acetate/hexane 3:1) 0.24; $[\alpha]^{25}$ - $104.7\left(c 1, \mathrm{CHCl}_{3}\right) ;{ }^{1} \mathrm{H} \mathrm{NMR}\left(300 \mathrm{MHz}, \mathrm{CDCl}_{3}\right) \delta_{\mathrm{H}}$ 7.37-7.11 (6H, m), $6.44(1 \mathrm{H}, \mathrm{d}, J=9.1 \mathrm{~Hz}), 6.01(1 \mathrm{H}, \mathrm{d}, J=6.5 \mathrm{~Hz}), 5.02(1 \mathrm{H}, \mathrm{d}, J=12.4 \mathrm{~Hz}), 4.97(1 \mathrm{H}, \mathrm{d}, J=12.3 \mathrm{~Hz}), 4.38-4.22$ $(2 \mathrm{H}, \mathrm{m}), 4.16(1 \mathrm{H}, \mathrm{d}, J=15.8 \mathrm{~Hz}), 3.86(1 \mathrm{H}, \mathrm{dd}, J=15.8,7.2 \mathrm{~Hz}), 3.18-2.96(3 \mathrm{H}, \mathrm{m}), 2.48-2.38(1 \mathrm{H}, \mathrm{br}, \mathrm{s}), 2.05-1.88$ $(2 \mathrm{H}, \mathrm{m}) ;{ }^{13} \mathrm{C}$ NMR $\left(75.4 \mathrm{MHz}, \mathrm{CDCl}_{3}\right) \delta_{\mathrm{C}} 163.4,155.3,148.8,138.6,136.3,127.8-128.5(5 \mathrm{C}), 117.4,105.4,67.4,50.0$, 51.1, 49.0, 34.5, 27.3, 25.8; HRMS calcd for $\mathrm{C}_{19} \mathrm{H}_{20} \mathrm{~N}_{2} \mathrm{O}_{3}$ : 324.3829. Found: 324.3837 .

\section{(-)-cytisine 1}

To a solution of 12 (110 mg, $0.34 \mathrm{mmol})$ in THF ( $6 \mathrm{~mL}), 6 \mathrm{~N} \mathrm{HCl}(2.2 \mathrm{~mL})$ was added and the solution was refluxed for $3 \mathrm{~h}$. Then water $(3 \mathrm{~mL})$ was added and THF was removed in vacuum. The aqueous phase was basified with $1 \mathrm{~N} \mathrm{NaOH}$ (pH 9-10) and extracted with diethyl ether. The combined organic extracts were dried over $\mathrm{Na}_{2} \mathrm{SO}_{4}$ and the solvent was evaporated to yield $50 \mathrm{mg}(78 \%)$ of $\mathbf{1}$, as an oil: $\mathrm{R}_{f}$ (ethyl acetate) 0.02 ; $[\alpha]^{25} \mathrm{D}-114.2(c 1, \mathrm{EtOH}) ;{ }^{1} \mathrm{H} \mathrm{NMR}(300 \mathrm{MHz}$, $\left.\mathrm{CDCl}_{3}\right) \delta_{\mathrm{H}} 7.26(1 \mathrm{H}, \mathrm{dd}, J=9.1,6.8 \mathrm{~Hz}), 6.41(1 \mathrm{H}, \mathrm{dd}, J=9.1,1.3 \mathrm{~Hz}), 5.88(1 \mathrm{H}, \mathrm{dd}, J=6.8,1.3 \mathrm{~Hz}), 4.10(1 \mathrm{H}, \mathrm{d}, J=$ $15.6 \mathrm{~Hz}), 3.86(1 \mathrm{H}, \mathrm{ddd}, \mathrm{J}=15.6,6.6,0.9 \mathrm{~Hz}), 3.07(1 \mathrm{H}, \mathrm{dd}, J=12.4,1.3 \mathrm{~Hz}), 3.04(1 \mathrm{H}, \mathrm{dd}, J=12.1,2.4 \mathrm{~Hz}), 2.99$ 
$(2 \mathrm{H}, \mathrm{br}, \mathrm{d}, J=12.3 \mathrm{~Hz}), 2.86(1 \mathrm{H}, \mathrm{br}, \mathrm{s}), 2.31(1 \mathrm{H}, \mathrm{br}, \mathrm{s}), 1.94(2 \mathrm{H}, \mathrm{t}, \mathrm{J}=3.2 \mathrm{~Hz}), 1.51(1 \mathrm{H}, \mathrm{s}) ;{ }^{13} \mathrm{C} \mathrm{NMR}(75.4 \mathrm{MHz}$, $\left.\mathrm{CDCl}_{3}\right) \delta_{\mathrm{C}} 163.7,151.1,138.8,116.8,104.9,54.00,53.00,49.7,35.6,27.8,26.3$; HRMS calcd for $\mathrm{C}_{11} \mathrm{H}_{14} \mathrm{~N}_{2} \mathrm{O}$ : 190.2470. Found: 190.2465. 